\title{
Comments: Improving Weighting Methods for Causal Mediation Analysis
}

\author{
Kosuke Imai \\ Princeton University, Princeton, New Jersey, USA
}

I begin this discussion by thanking Larry Hedges, the editor of the journal, for giving me an opportunity to provide a commentary on this stimulating article. I also would like to congratulate the authors of the article for their insightful discussion on causal mediation analysis, which is one of the most important and challenging methodological problems we face in the literature of causal inference. I particularly admire the authors' efforts to clearly explain complex statistical concepts in the context of their specific application. I have no doubt that applied education researchers who read this article will gain a better understanding of important methodological issues regarding causal mediation analysis.

Although there are many positive things to be said about the authors' proposed methodology, in this commentary I discuss one potential way to further improve it. In particular, the authors argue that the proposed ratio-of-mediator-probability weighted (RMPW) estimation methodology is attractive because unlike some of the existing methods it "does not involve explicit modeling of the mediator-outcome relationship" (p. 273). Although this is clearly an advantage, the RMPW estimation methodology still requires researchers to correctly model the mediator given the treatment and pretreatment confounders. This is often a challenging task because there may exist a large number of pretreatment confounders. Because nonparametric modeling in a high-dimensional covariate space is difficult, these confounders often must be adjusted through a parametric model.

A similar problem applies to propensity score methods. For example, Kang and Schafer (2007) showed that slight misspecification of propensity score model can yield a large bias in the estimation of treatment effects. They found that the magnitude of such bias can be quite substantial for propensity score weighting methods when the severe selection bias leads to small weights. Imai King and Stuart (2008) called this problem "propensity score tautology"- propensity score methods allow researchers to avoid the modeling of the outcome-treatment relationship only when they can correctly model the treatment-covariate relationship. The proposed RMPW estimation methodology resembles propensity score weighting methods in that researchers must confront the equally difficult task of correctly modeling the conditional distribution of mediator given the treatment and pretreatment confounders.

To address this issue, Imai and Ratkovic (2012) proposed the covariate balancing propensity score (CBPS) estimation as the robust parametric estimation strategy for the propensity score. The idea is to exploit the dual characteristics of the propensity score as the conditional probability of treatment assignment and a balancing score: If the propensity score is correctly estimated, it should predict the treatment assignment $Z$ and balance the covariate distribution between the treatment and control groups (once the covariates $X$

Address correspondence to Kosuke Imai, Department of Politics, Princeton University, Princeton NJ 08544, USA. E-mail: kimai@ princeton.edu 
are appropriately weighted via the inverse of the propensity score). Specifically, Imai and Ratkovic proposed to use the Generalized Method of Moments (GMM) estimation with the following overidentifying moment conditions,

$$
\begin{aligned}
\mathbb{E}\left\{\frac{Z \pi_{\theta}^{\prime}(X)}{\pi_{\theta}(X)}-\frac{(1-Z) \pi_{\theta}^{\prime}(X)}{1-\pi_{\theta}(X)}\right\} & =0 \\
\mathbb{E}\left\{\frac{Z \tilde{X}}{\pi_{\theta}(X)}-\frac{(1-Z) \tilde{X}}{1-\pi_{\theta}(X)}\right\} & =0
\end{aligned}
$$

where $\tilde{X}$ is a vector-valued function of $X$ selected by the researcher, $\pi_{\theta}(X)=\operatorname{Pr}_{\theta}(Z=1$ । $X)$ is the propensity score with a vector of unknown parameters $\theta$, and $\pi_{\theta}^{\prime}(X)=\frac{\partial}{\partial \theta^{\top}} \pi_{\theta}(X)$ is its first derivative with respect to $\theta$. Equation 1 represents the score function derived from the likelihood function, corresponding to the fact that the propensity score is defined as the conditional probability of treatment assignment. The moment condition given in Equation 2, on the other hand, represents the covariate balancing condition where some functions of $X$ are balanced in expectation. Thus, what the CBPS estimation accomplishes is to estimate the propensity score such that the covariate balance is optimized. Imai and Ratkovic (2012) found that the resulting CBPS dramatically improves the empirical performance of propensity score weighting methods and overcomes the critiques of Kang and Schafer (2007).

I conjecture that the CBPS estimation can improve the performance of the proposed RMPW estimation methodology. As discussed in Imai and Ratkovic (2012), the CBPS can be easily extended to nonbinary treatment regimes, which implies that it can be applied to the RMPW estimation even when the mediator is not binary. In particular, instead of fitting the ordered logistic regression via maximum likelihood as suggested by the authors, we can use the GMM estimation based on the following moment conditions. First, we have a set of moment conditions representing the score function from the likelihood,

$$
\mathbb{E}\left\{\sum_{c=0}^{3} \frac{\mathbf{1}\{C=c\}}{\pi_{\theta}^{c}(V)} \cdot \frac{\partial \pi_{\theta}^{c}(V)}{\partial \theta^{\top}}\right\}=0
$$

where $\pi_{\theta}^{c}(V)=\operatorname{Pr}_{\theta}(C=c \mid V)$ is the propensity score for the mediator level $c \in$ $\{0,1,2,3\}, V=(Z, X, \bar{X}, W(1), W(0))$, and $\theta=\left(\beta_{1}, \beta_{2}, \beta_{3}, \beta_{4}, d_{1}, d_{2}, d_{3}, \tau\right)$. Second, we have the set of covariate balancing moment conditions,

$$
\mathbb{E}\left\{\frac{\mathbf{1}\{C=c\} V}{\pi_{\theta}^{c}(V)}-\frac{\mathbf{1}\{C=c-1\} V}{\pi_{\theta}^{c-1}(V)}\right\}=0
$$

for $c=1,2,3$. Thus, without modifying the model used for the conditional distribution of the mediator, the CBPS estimation provides a robust way of estimating the weights for the proposed RMPW methodology.

Finally, although the improvement of the estimation techniques such as the one described above is possible, I emphasize that like the existing methodologies, the RMPW estimation methodology relies upon strong identification assumptions that are not directly testable from the observed data. In particular, these methods require that there exist no posttreatment confounders, whether observed or unobserved, for the relationship between the mediator and the outcome. This is a strong assumption because in many cases there 
are multiple mediators that are causally related to each other. In my opinion, there are two ways to address this important methodological problem. First, one can conduct sensitivity analyses within a certain modeling framework. For example, Imai and Yamamoto (2011) developed a sensitivity analysis using a semiparametric random coefficient linear regression model. Similarly, Tchetgen Tchetgen and Shpitser (2011) proposed a sensitivity analysis under a different semiparametric model. Second, researchers can utilize alternative research designs so that strong assumptions can be relaxed. Imai, Tingley, and Yamamoto (2012) and Mattei and Mealli (2011) developed several such experimental designs. These recent developments are important because they directly address the limitations of the standard causal mediation analysis. Therefore, I conclude this discussion by encouraging the authors and others to make future methodological contributions to these promising new research agendas about causal mediation analysis.

\section{ACKNOWLEDGMENTS}

The author acknowledges final support by the National Science Foundation (SES-0918968).

\section{REFERENCES}

Imai, K., King, G., \& Stuart, E.A. (2008). Misunderstandings among experimentalists and observationalists about causal inference. Journal of the Royal Statistical Society, Series A (Statistics in Society), 171, 481-502.

Imai, K., \& Ratkovic, M. (2012). Covariate balancing propensity score (Working paper). Available at from http://imai.princeton.edu/research/CBPS.html

Imai, K., Tingley, D., \& Yamamoto, T. (2012). Experimental designs for identifying causal mechanisms (with discussions). Journal of the Royal Statistical Society, Series A (Statistics in Society). Forthcoming.

Imai, K., \& Yamamoto, T. (2011). Identification and sensitivity analysis for multiple causal mechanisms: Revisiting evidence from framing experiments (Working paper). Available from http://imai.princeton.edu/research/medsens.html

Kang, J. D., \& Schafer, J. L. (2007). Demystifying double robustness: A comparison of alternative strategies for estimating a population mean from incomplete data (with discussions). Statistical Science, 22, 523-539.

Mattei, A., \& Mealli, F. (2011). Augmented designs to assess principal strata direct effects. Journal of the Royal Statistical Society, Series B (Methodological), 73, 729-752.

Tchetgen Tchetgen, E. J., \& Shpitser, I. (2011). Semiparametric theory for causal mediation analysis: Efficiency bounds, multiple robustness, and sensitivity analysis (Tech. rep.). Harvard University School of Public Health, Cambridge, MA. 